\title{
Clinical Features in Predicting COVID-19
}

\author{
Balzanelli GM ${ }^{1 *}$, Distratis $\mathrm{P}^{1 *}$, Aityan $\mathrm{KS}^{2}$, Amatulli $\mathrm{F}^{1}$, Catucci $\mathbf{0}^{1}$, Cefalo $\mathrm{A}^{1}$, D'Angela $\mathrm{G}^{1}$, Dipalma $\mathrm{G}^{3}$, \\ Inchingolo $\mathrm{F}^{3}$, Lazzaro $\mathrm{R}^{1}$, Nguyen $\mathrm{KCD}^{4}$, Palazzo $\mathrm{D}^{1}$, Pham $\mathrm{HV}^{5}$, Tomassone $\mathrm{D}^{6}$, Tran Cong $\mathrm{T}^{7 *}$ and \\ Gargiulo Isacco $\mathrm{C}^{1,3,4 *}$ \\ ${ }^{1}$ SET-118, Department of Pre-hospital and Emergency SG Giuseppe Moscati Hospital, Taranto City, 74100, Italy \\ ${ }^{2}$ Department of Multidisciplnary Research Centre, Lincoln University, Oakland CA, 94612, USA
}

3"Aldo Moro" Unversity of Bari School of Medicine D.I.M. (Department of Interdisciplinary Medicine), Bari City, 70125, Italy

${ }^{4}$ American Stem Cells Hospital and Human Stem cells Institute, Ho Chi Minh City, 70000, Vietnam

${ }^{5}$ Phan Chau Trinh University of Medicine and Nam-Khoa Biotek, Ho Chi Minh City, 50000, Vietnam

${ }^{6}$ Nutritherapy Research Center, Urbino City, 10098, Italy

${ }^{7}$ Pham Ngoc Thach University of Medicine, Department of Histology, Embryology and Genetics, Ho Chi Minh City, 70000, Vietnam

*Corresponding author: Balzanelli GM, Distratis P, SET-118, Department of Pre-hospital and Emergency - SG Giuseppe Moscati Hospital, Taranto City, 74100, Italy

Tran Cong Toai, Pham Ngoc Thach University of Medicine, Department of Histology, Embryology and Genetics, Ho Chi Minh City, 70000, Vietnam

Gargiulo Isacco, "Aldo Moro" University of Bari - School of Medicine D.I.M. (Department of Interdisciplinary Medicine), Bari City, 70125, Italy, American Stem Cells Hospital and Human Stem cells Institute, Ho Chi Minh City, 70000, Vietnam, SET-118, Department of Pre-hospital and Emergency - SG Giuseppe Moscati Hospital, Taranto City, 74100, Italy

\section{ARTICLE INFO}

Received: 慧 August 11, 2020

Published: 幽 August 31, 2020

Citation: Balzanelli G M, Distratis P, Aityan KS, Amatulli F, Catucci O, Cefalo A, et al. Clinical Features in Predicting COVID-19. Biomed J Sci \& Tech Res 29(5)-2020. BJSTR. MS.ID.0048743.

Keywords: SG Moscati Hospital; 118 Pre-Hospital Medical System-Emergency Department; COVID 19, Sars-CoV-2; Arterial Blood Gas (ABG); Trojan Horse; Bronchio-Alveolar Lavage Fluid (BALF)

\section{ABSTRACT}

The coronavirus COVID-19 disease is being a hard task for emergency care units worldwide due to the uncharacteristic disease course consequently to the scarcity of information and medical resources. The SARS-CoV-2 is a member of the betacoronavirus genus, which includes the SARS-CoV coronavirus, the MERS coronavirus, and two other human coronaviruses, HCoV-OC43 and HCoV-HKU1. Compared to SARS-CoV and MERS coronaviruses SARS-CoV-2 has shown a higher degree of contagiousness. The degree of lung damages caused by SARS-CoV-2 infection appeared to be dissimilar to the patient's prognosis and with the grade of systemic severity presented by clinical manifestations at first admission into the 118 Pre-Hospital and Emergency department. Clinically, a consistent number of patients with mild symptoms on the arrival developed an unexpected clinical worsening manifesting an acute respiratory distress syndrome during the next hours from the admission time. Therefore, it was essential to rapidly recognize the extent of the ongoing disease progression before either thoracic CT-scan or nasopharyngeal swab RT-PCR test, as both need time before being applied or giving a result. Thus the primary purpose of this article was to predict COVID-19 advancement by using Arterial Blood Gas (ABG), laboratory, and CBC analysis as a valuable tool for the preliminary prognostic assessment of patients with SARS-CoV-2 infection accepted into the emergency department. 


\section{Introduction}

The first cases of COVID-19 in Italy were confirmed on February 21st, 2020 in Codogno and Vo' (in the northen part of Italy). From March 27th 2020 up to May 25th (the considered period), a total of 381 patients were admitted to our 118 Emergency Department in SG Moscati Hospital of Taranto City, most of whom were male [n= 229[61\%]), 19 deceased (2 in 118 Department, 5 in Pneumology Department, 5 in Intensive Care Unit (ICU) and 7 in Infectious Disease Department [n=19 [5\%]) over $80 \%$ of older age (mean age 72 years). The 118 Pre-Hospital Medical System Emergency operating in the province of Taranto in Italy received 1008 calls, in the period from February 24th 2020 up to May 12th 2020, for patients who reported symptoms attributable to possible respiratory infections or who accused to have contact with people with suspected or acclaimed SARS-CoV-2 infection. 40,96\% of calls were for female patients while $59,04 \%$ were for male patients.

The symptoms they reported were: fever (77,88\%), cough $(42,46 \%)$, malaise $(39,98 \%)$, dyspnoea $(36,51 \%)$, headache $(23,71 \%)$, cold $(18,15 \%)$, sore throat $(16,67 \%)$, conjunctivitis $(14,48 \%)$, changes in taste and / or smell $(13,09 \%)$, gastrointestinal symptoms (8,04\%); 9,03\% of them had a contact with a sure SARSCoV-2 infection patients; 3,17 \% had an asymptomatic SARS-CoV-2 infection. The patients reporting the symptoms were divided into these age groups: 0-9 years (1,70\%), 10-19 years (3,80\%), 20-34 years $(13,19 \%), 35-59$ years $(36,66 \%), 60-69$ years $(14,29 \%)$, $70-79$ years $(15,08 \%), 80-89$ years $(11,99 \%), \geq 90$ years $(3,30$ $\%)$. The abnormal ABG profile was characteristics of an acute respiratory hypoxemic, hypocapnic state with a compesatory alkalosis, suggestive of a progressive pulmonary micro-embolism revealing an internal, persistent hypercoagulable process with endothelial activation as consequence of an uncotrolled increase of proinflammatory cytokines [1].

The body's response to the hypoxaemic state, is described by an increase in minute ventilation that drives to an unrestrainable hypocapnia, as the $\mathrm{CO}_{2}$ diffuses through tissues about 20 times faster than $\mathrm{O}_{2}$. Though the pathoanatomical and pathophysiological basis for respiratory failure in COVID-19 remains unclear, the presence of progressive and diffuse tissue and alveolar damages with interstitial thickening and thromboembolisms and gas exchange impairments seem to be the reasonable mechanism. A scenario that is often accompanied with atelectasis and lung consolidations clearly seen in CT-scan images with typical ground glass opacities $[2,3]$. Nevertheless, the combination of ABG analysis, (the oxygen saturation level $\left(\mathrm{PaO}_{2}\right)$ and the CT-scan revealed to be a better tool in diagnosting COVID-19 than swab RT-PCR alone (total patients screened=310). Within the range of $\mathrm{PaO}_{2}$ up to $60 \mathrm{mmHg}$, on total of screened 59 patients the CT confirmed 27 positive case versus only 11 positive cases revealed by swab RT-PCR (Table 1, column 1); with a $\mathrm{PaO}_{2}$ between 61 to $70 \mathrm{mmHg}$, on a total of 64 patients, CT confirmed 20 positives versus 12 positives confirmed by RT-PCR (Table 1, column 2); with a $\mathrm{PaO}_{2}$ between 71 to $90 \mathrm{mmHg}$, on a total of 132 patientss, the CT confirmed 22 positives versus 17 positives confirmed by RT-PCR (Table 1, column 3); with a $\mathrm{PaO}_{2}$ within 90 mmHg, with a total number of 55 patientss, the CT confirmed 5 positive cases versus just 2 positives revealed by RT-PCR (Table 1, column 4).

Table 1: The ABG parameters were indicative in presence of COVID-19 infection. The $\mathrm{pH}$, the $\mathrm{paO}_{2}$ and paCO $\mathrm{C}_{2}$ measured in the group of Pos-Pos (swab positive and CT scan positive) were remarkably abnormal in presence of Sars-CoV-2 pneumonia.

\begin{tabular}{|c|c|c|c|c|}
\hline & Total & pH & $\mathrm{PaO}_{2}$ & $\mathrm{PaCO}_{2}$ \\
\hline N.\% & Patients & $(>7.45)$ & $(<75)$ & $(<35)$ \\
\hline POS-POS & $\begin{array}{c}46 \\
\text { M } 34=74 \% \\
\text { F } 12=26 \%\end{array}$ & $\begin{array}{c}33=72 \% \\
\text { M } 26=57 \% \\
\text { F } 7=15 \%\end{array}$ & $\begin{array}{c}33=72 \% \\
\text { M } 27=59 \% \\
\text { F 6 }=13 \%\end{array}$ & $\begin{array}{c}33=72 \% \\
\text { M 26 }=57 \% \\
\text { F 7 }=15 \%\end{array}$ \\
\hline
\end{tabular}

Table 2: Covid-19 admitted patients ( $n=310)$ into 118 Emergency Department were screened for arterial blood gas (ABG) analysis, Swab Buffer (oral-nasal-pharinx) analyzed by RT-PCR and thoracic CT scan were then performed and results were compared. Notably, based on the oxygen saturation level $\left(\mathrm{pO}_{2}\right)$ the CT scan revealed, in line with different published data, a better degree of accuracy compared to swab-RT-PCR outcomes, the $\mathrm{PaO}_{2}$ up to $60 \mathrm{mmHg}$ on total of 59 patients the CT+ was $27 \mathrm{vs} 11 \mathrm{swab}+($ column 1); the $\mathrm{PaO}_{2}$ from 61 to $70 \mathrm{mmHg}$, total 64 cases, $20 \mathrm{CT}+\mathrm{vs} 12 \mathrm{swab}+\left(\right.$ column 2); the $\mathrm{PaO}_{2}$ from 71 to $90 \mathrm{mmHg}$, total $132 \mathrm{cases}, 22$ $\mathrm{CT}+$ vs 17 swab+ (column 3); the $\mathrm{PaO}_{2} 90 \mathrm{mmHg}$, total 55 cases, $5 \mathrm{CT}+\mathrm{vs} 2 \mathrm{swab}+$ (column 4).

\begin{tabular}{|c|c|c|c|}
\hline$\left(\mathrm{PaO}_{2}\right)$ & $\left(\mathrm{PaO}_{2}\right)$ & $\left(\mathrm{PaO}_{2}\right)$ & $\left(\mathrm{PaO}_{2}\right)$ \\
\hline up to $60 \mathrm{mmHg}$ & 61 to $70 \mathrm{mmHg}$ & 71 to $90 \mathrm{mmHg}$ & $>90 \mathrm{mmHg}$ \\
\hline Patients n. 59 & Patients n. 64 & Patients n. 132 & Patients n. 55 \\
\hline 11 Swab + & 12 Swab + & 17 Swab + & $2 \mathrm{Swab}+$ \\
\hline 48 Swab - & 52 Swab - & 115 Swab - & 53 Swab - \\
\hline CT scan & CT scan & CT scan & CT scan \\
\hline $27 \mathrm{CT}+$ & $20 \mathrm{CT}+$ & $22 \mathrm{CT}+$ & $5 \mathrm{CT}+$ \\
\hline 32 CT - & 44 CT - & 110 CT - & $50 \mathrm{CT}$ - \\
\hline
\end{tabular}


The cell blood count ( $\mathrm{CBC}$ ) and microbiology analysis was ectremely important in confirming a progressive systemic decay. The COVID-19 patients's CBC performed immediately after the ABG tests, was suggestive of an uncontrolled infectious-inflammatory condition with multi-organ involvement particularly reffered to lungs, heart and kidneys. A significative number of patients revealed a high level of total white cell count (WBC > 10.000 cells $/ \mathrm{mcL}$ ), with neutrophilia and marked lymphopenia. Laboratory outcomes confirmed kidney, heart and liver involvement with low level of eGFR and $250 \mathrm{H}$-vitamin D, increased level of troponin, IL-6, D-Dimer and ESR and an augmented level of fibrinogen (Tables 3-6). The ensuing infectious phase was contradistinguished by an unarrestable hyper-inflammatory cascade chracterized by the formation of multiple thrombi and lesions at the level of microvascular vessels with a generalized compromised endothelial tissue. This phase was contradistinguished by a secondary aggressive bacterial infection with an inflating progression that became clinically and symptomatically evident. In the worst cases, the rapidity of multiorgan involvement with contextual septic course was often related to the contribution of different pathogens identified in BALF and blood culture, such as Klebsiella spp, Candida albicans, Aspergillus, Pseudomonas spp, almost a prerogative feature of the conclusive phase of SARS-CoV-2 [2-6].

Table 3: The lymphocyte parameter in CBC was indicative in presence of COVID-19 infection. The lymphopenia detected in the group of Pos-Pos (swab positive and CT scan positive) was suggestive of Covid-like disease.

\begin{tabular}{|c|c|c|c|c|}
\hline & Total & Neutr. \% & Lymph. \% & WBC \% \\
\hline N.\% & Patients & $(>75)$ & $(<20)$ & $(>10)$ \\
\hline POS-POS & $\begin{array}{c}46 \\
\text { M } 34=74 \% \\
\text { F } 12=26 \%\end{array}$ & $\begin{array}{c}27=59 \% \\
\text { M } 21=46 \% \\
\text { F } 6=13 \%\end{array}$ & $\begin{array}{c}35=76 \% \\
\text { M } 28=61 \% \\
\text { F 7 }=15 \%\end{array}$ & $\begin{array}{c}16=35 \% \\
\text { M 13 }=28 \% \\
\text { F } 3=6 \%\end{array}$ \\
\hline
\end{tabular}

Table 4: The eGFR parameter in kidney functionality, the range between 60-90 assessed in CBC was significative in the presence of COVID-19 infection. The lymphopenia detected in the group of Pos-Pos (swab positive and CT scan positive) was remarkably higher compared with the other two groups Covid-like or Neg-Pos Neg-Pos (swab negative and CT scan positive), Neg-Neg (swab negative and CT scan negaitive). The data confirmed that COVID-19 could be a gender specific diseass. Male patients were consistently higher in Pos-Pos and Covid-like Neg-Pos vs Neg-Neg group (blue-highlight).

\begin{tabular}{|c|c|c|c|c|}
\hline & Total & eGFR \% & eGFR \% & eGFR \% \\
\hline N.\% & Patients & $\mathbf{( < 6 0 ~} \mathbf{~ m l} / \mathbf{m i n})$ & $\mathbf{( 6 0 - 8 9 ~} \mathbf{~ m l} / \mathbf{m i n})$ & $\mathbf{( > 9 0} \mathbf{~ m l} / \mathbf{m i n})$ \\
\hline \multirow{2}{*}{ POS-POS } & 46 & $14=31 \%$ & $22=48 \%$ & $10=21 \%$ \\
& F $12=26 \%$ & F $4=8 \%$ & F $5=11 \%$ & F $2=4 \%$ \\
M $34=74 \%$ & M $10=23 \%$ & M $17=37 \%$ & M $8=17 \%$ \\
\hline
\end{tabular}

Table 5: The CRP (C-Reactive Protein) and ESR (Erythrocyte Sedimentation Rate) parameters the range assessed in CBC was significative high in presence of COVID-19 infection.

\begin{tabular}{|c|c|c|c|c|}
\hline & Total & ESR & CRP & D-dimer \\
\hline N.\% & Patients & $\mathbf{( > 1 0 ~} \mathbf{m m} / \mathbf{h})$ & $\mathbf{( > 3 . 5 ~} \mathbf{~ m g} / \mathbf{L})$ & (>0.5 $\mathbf{~ m g} / \mathbf{L})$ \\
\hline \multirow{2}{*}{ POS-POS } & 46 & $43=93 \%$ & $46=100 \%$ & $17=37 \%$ \\
& F $12=26 \%$ & F $11=24 \%$ & F $12=26 \%$ & F $6=13 \%$ \\
& M 34=74\% & M 32 $=70 \%$ & M 34=74\% & M $11=24 \%$ \\
\hline
\end{tabular}

Table 6: The lymphocyte parameter in CBC was indicative in presence of COVID-19 infection. The lymphopenia detected in the group of Pos-Pos (swab positive and CT scan positive) was suggestive of Covid-like disease.

\begin{tabular}{|c|c|c|c|}
\hline & Total & Fibrinogen & Troponin \\
\hline N.\% & Patients & $\mathbf{( > 4 0 0 ~} \mathbf{~ m g} / \mathbf{d L})$ & $\mathbf{( > 1 4} \mathbf{~ n g} / \mathbf{L})$ \\
\hline \multirow{2}{*}{ PoS-POS } & 46 & $44=96 \%$ & $14=30 \%$ \\
& F 12=26\% & F $11=24 \%$ & M $3 \%$ \\
M 34=74\% & M 33=72\% & M $11=24 \%$ \\
\hline
\end{tabular}

\section{Prognostic Factors of Severe Illness}

The pathogenic mechanism that produces pneumonia seems to be particularly complex, especially with regard to the infection progression which eventually evolves into compromised multi-organ disease. At the moment, the data available seem to indicate a Sars-CoV-2 as the main pathogen capable of triggering the entire immune chain reaction that takes place with an increasing intensity within not only within the broncho-alveolar interstices but to surrounding tissues and organs as well. The induced endothelitis has been seen as major causative factors that affected microcirculatory activity with the insurgence of the "happy hypoxia", a phenomena described by J. Couzin-Frankel [7]. According to the author, the tissue necrosis should be linked to a generalized augmentation of inflammatory processes co- 
existing with a progressive state of infection driving to microvascular thrombosis that is often carachterized by a non-specific suggestive lymphopenia that may substantially refer to a dangerassociated molecular pattern predictive of an auto-generating necroinflammatory loop arrangement [7-10]. The unexpected worsening of the entire condition that eventually resolved into a massive and disseminated intravascular coagulation (DIC), deep intravascular thrombotic processess and sepsis syndrome has been generally accepted as typical clinical trait of COVID-19 affected patients (Figures 1\& 2) [11-15].

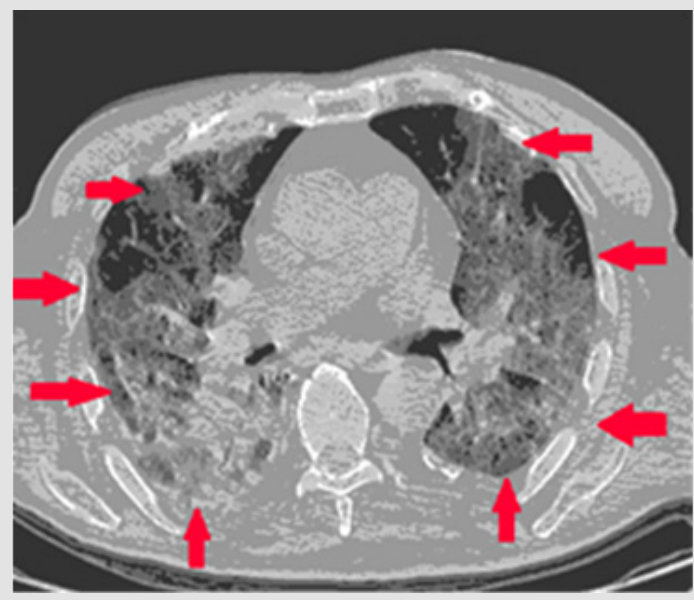

Figure 1: Unenhanced CT images in a 33-year-old woman. A, Image shows multiple ground-glass opacities in bilateral lungs. Ground-glass opacities with reticulations in a peripheral distribution. Pleural effusion and pericardium effusion were localized mediastinally. Findings were consistent with Covid-19 pneumonia. The bilateral opacities localized peripherally with subpleural "spider-like net" (red arrows) are common CT findings of COVID-19 pneumonia.

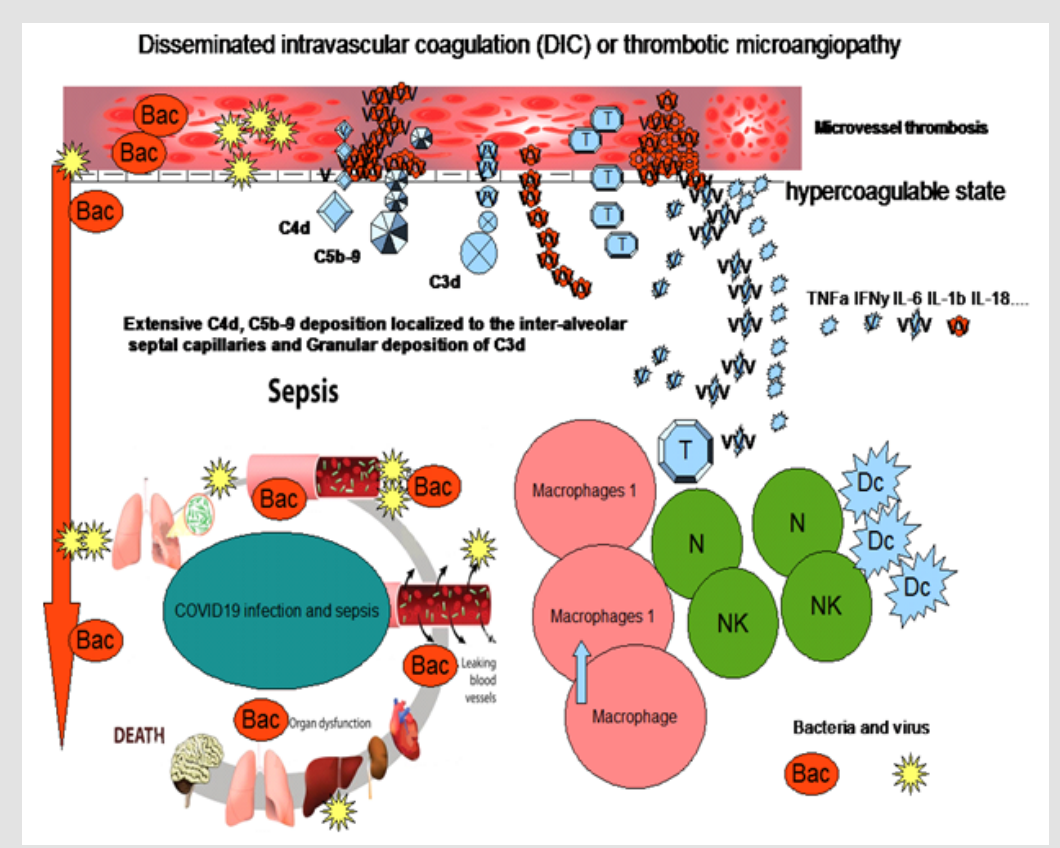

Figure 2: An algorithm to clearly understand the disseminated intravascular coagulation (DIC), the intravascular thrombotic process (ITP) and sepsis syndrome (SS) in COVID-19 patients admitted in our Hospital. Thrombocytic evoltutive mechanism associated with high level of pro-inflammatory cytokines and interleukins, macrophage, T cells, DCs, NK and neutrophils hyper responses with scattering presence of both bacteria and virus lead to a progressive organ dysfunction and sepsis. Abnormal coagulation process, fibrinogen concentration and D-dimers are evident part of this mechanism and revealed to be a key factor for the differentiation of DIC from thrombotic microangiopathies (TMAs). Once DIC has been proved, the underlying superinflammatory process with multiple tissue and organ disseminated infection must be identified. This would be diagnosed by the identification of low eGFR, high level of troponin and fibrinogen together with high levels of ESR and D-Dimer associated with an increased level of neutrophils and a low level of lymphocytes. Positive netrophilia and lymphopenia, with high level of IL-6 strongly suggests a deteriorating DIC with an associated complement-activating condition like infection and progressive multi-organ failure [16-23] (Ciro Gargiulo, Rita Lazzaro, Mario Balzanelli). 
Descriptive statistics were used to summarize the data; results are reported as medians ranges as appropriate. Categorical variables were summarized as counts and percentages. Statistical analysis was supported by Student-t test. The null hypotheswas HO was calculated by using t-statistics. Preliminary results from this study suggested that the ABG test performed and CBC analysis upon admission of patients with suspected COVID-19 in the 118 Emergency Department may be suggestive of the extent of lung inflammatory/infectious process providing warnings for the patient's prognosis. The obtained results from ABG analysis measured at this time, performed before CT scan image and swab RTPCR, indicated that 33 patients on a total of 46 confirmed COVID-19 (72\%) revealed alkalosis, hypocapnia and hypoxia $(\mathrm{pH}>7.45$; low $\mathrm{PaO}_{2}<75$; low $\mathrm{PaCO}_{2}<35$ ) (Table 1) and 46 on 46 showed CT-scan with ground glass opacities (significance level 0.05). These results were in line with findings observed in precedent studies, in which a higher percentage of the lung being involved in the inflammatory process, as then presented on the CT scan images that appeared to correlate with decreased oxygenation capacity [16].

This clinical picture appeared to emphasize the severity of the patient's disease progression. Reduced ABG values and abnormal CBC and microbiology parameters into 118 Emergency Department arrival may be suggestive of greater essential severity of the disease process and allowing to recognize who eventually present with a subtle unfavorable condition even if not clinically compromised [16]. A quantitative assessment allowed us to assess in a predictable manner and with a certain precision the progression of the SarsCoV-2 infection. The current study and data were obtained on a group of confirmed COVID-19 patients divided according to ABG, CT scan and RT-PCR results We were able to assess significative markers that could characterize the COVID-19 infection allowing better future prognosis and prediction. These specific features were reported as follow (Table 7):

Table 7: We assume that, in doubtful situations, for instance, waiting for the swab result (the first or the second one), the combination of the above parameters might help to identify the risk of having COVID-19.

\begin{tabular}{|c|c|c|c|c|c|c|c|c|c|c|}
\hline \multirow{2}{*}{ RISK } & \multirow{2}{*}{ CT scan } & pH & $\mathrm{PaO}_{2}$ & $\mathrm{PaCO}_{2}$ & Lymph. \% & eGFR \% & Fibrinogen & ESR & CRP & \multirow{2}{*}{ SEX } \\
\hline & & $(>7.45)$ & $(<75)$ & $(<35)$ & $(<20)$ & $(60-89)$ & $(>400)$ & $(>10)$ & $(>3.5)$ & \\
\hline High & + & + & + & + & + & + & + & + & + & $\mathrm{M} / \mathrm{F}$ \\
\hline Medium & + & $+/-$ & $+/-$ & $+/-$ & + & $+/-$ & + & + & + & $M / F$ \\
\hline Low & - & - & - & - & $+/-$ & - & $+/-$ & + & + & M \\
\hline
\end{tabular}

a) The sex, male patients resulted higher in number with higher affected rate than females.

b) The compromised $\mathrm{ABG}$ profile, characterized by a marked hypoxia, hypocapnia and alkalosis at the time of the admission, revealing either low $\mathrm{PaO}_{2}$ saturation or low $\mathrm{PaCO}_{2}$ strongly suggestive of COVID-19 even before the RT-PCR and CT-scan procedure.

c) The lymphopenia was seen also a higher specific COVID-19 marker.

d) Significant high level of fibrinogen accompanied by a corresponding high level of CRP, ESR and the e-GFR (between 60 to 89 ) were the other specific parameters that revealed COVID-19 positivity.

In general, though the CT scan and RT-PCR are considered to be categorical markers of COVID-19 diagnosis, in our opinion this would be an error due to their non-specificity towards the diseases itself. Said that, an extensive lung infection confirmed by an ABG analysis indicating an alkalotic, hypocapnic and hypoxic state accompanied by a "ground glass opacity" CT images could be more suggestive for an immediate hospitalization in specific departments.

\section{Conclusion}

The results of this study suggest that in COVID-19 patients, the ABG test showed to be an important tool in evaluating and assessing the Sars-CoV-2 risk progression due to its rapidity and applicability. Due to the high risk of having a sudden worsening of the disease, we would suggest the physicians to act as soon as the first symptoms appear and move the patients to the nearest COVID Hospital. Worrisome signs would be a persistent low fever in a range between $37,0^{\circ}$ to $37,5^{\circ} \mathrm{C}$, severe and persistent dry cough, increasing dyspnoea, with an $\mathrm{ABG}$ result showing alkalosysis, low $\mathrm{PaO}_{2}$ and $\mathrm{PaCO}_{2}$. We are well aware that further studies are needed mainly related to the poor information regarding COVID-19. In addition, we also know that the relatively small number of the involved patients could eventually weaken the solidity of statistical analysis. Nevertheless, this model has been shown to be functional to the diverse application in different clinical scenarios of COVID-19, especially in supporting treatment decisions.

\section{Conflicts of Interest}

The authors whose names are listed above report the details of affiliation in an organization and entity with non-financial interest in the subject matter and materials discussed in this manuscript. 


\section{Ethical Approval}

This study was approved by the local ASL ethical committee.

\section{References}

1. Zhang Y, Xiao M, Zhang S, Peng Xia, Wei Cao, et al. (2020) Coagulopathy and antiphospholipid antibodies in patients with COVID-19. N Engl J Med.

2. Ottestad W, Søvik S (2020) COVID-19 patients with respiratory failure: what can we learn from aviation medicine? Br J Anaesth S00070912(20)30226-30229.

3. Tian S, Hu W, Niu L, Huan Liu, Haibo Xu, et al. (2020) Pulmonary pathology of early-phase 2019 novel coronavirus (COVID-19) pneumonia in two patients with lung cancer. J Thorac Oncol Adv 15: 700-704.

4. Yuen KS, Ye ZW, Fung SY, Chi-Ping Chan, Dong-Yan Jin (2020) SARS-CoV-2 and COVID-19: The most important research questions. Cell Biosci. 10: 40.

5. Lei J, Kusov Y, Hilgenfeld R (2018) Nsp3 of coronaviruses: Structures and functions of a large multi-domain protein. Antiviral Res 149: 58-74.

6. Nogueira MF, Pereira L, Jenull S, Karl Kuchler (2019) Klebsiella pneumoniae prevents spore germination and hyphal development of Aspergillus species. Sci Rep. 9(1): 218.

7. Couzin Frankel J (2020) The mystery of the pandemic's 'happy hypoxia'. Science. 368: 455-456.

8. Tan L, Wang Q Zhang D, Jinya Ding, Qianchuan Huang, et al. (2020) Lymphopenia predicts disease severity of COVID-19: a descriptive and predictive study. Sig Transduct Target Ther 5: 33.

\section{ISSN: 2574-1241}

DOI: 10.26717/BJSTR.2020.29.004873

Balzanelli G M. Biomed J Sci \& Tech Res

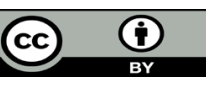

This work is licensed under Creative Commons Attribution 4.0 License

Submission Link: https://biomedres.us/submit-manuscript.php
9. Zhu N, Zhang D, Wang W, Xingwang Li, Bo Yang, et al. (2020) A Novel Coronavirus from Patients with Pneumonia in China 2019. N Engl J Med. 382: 727-733.

10. Belen-Apak FB, Sarialioglu F (2020) Pulmonary intravascular coagulation in COVID-19: possible pathogenesis and recommendations on anticoagulant/thrombolytic therapy. J Thromb Thrombolysis 1-3.

11. Moore H, Barrett CD, Moore EE, Robert C McIntyre, Peter K Moore, et al. (2020) Is there a role for plasminogen activator (tPA) as a novel treatment for refractory COVID-19 associated with acute respiratory distress syndrome (ARDS)? J Trauma Acute Care Surg. 88(6): 713-714.

12. Xu Z, Shi L, Wang Y, Jiyuan Zhang, Lei Huang, et al. (2020) Pathological findings of COVID-19 associated with acute respiratory distress syndrome. Lancet Respir Med. 8: 420-422.

13. Pan F, Ye T, Sun P, Shan Gui, Bo Liang, et al. Time Course of Lung Changes at Chest CT during Recovery from Coronavirus Disease 2019 (COVID-19). Radiology. 295(3): 715-721.

14. Bernheim A, Mei X, Huang M, Yang Yang, Zahi A Fayad, et al. (2020) Chest CT Findings in Coronavirus Disease-19 (COVID-19): Relationship to Duration of Infection. Radiology. 295(3): 200463.

15. Conway SE, Roy-O’Reilly M, Friedler B, Staff I, Furtunato G, et al. Sex differences and the role of IL-10 in ischemic stroke recovery. Biology of sex differences 6: 17 .

16. Turcato G, Panebianco L, Zaboli A, Christoph Scheurer, Dietmar Ausserhofer, et al. (2020) Correlation between arterial blood gas and CT volumetry in patients with SARS-CoV-2 in the emergency department. International Journal of Infectious Diseases 97: 233-235.

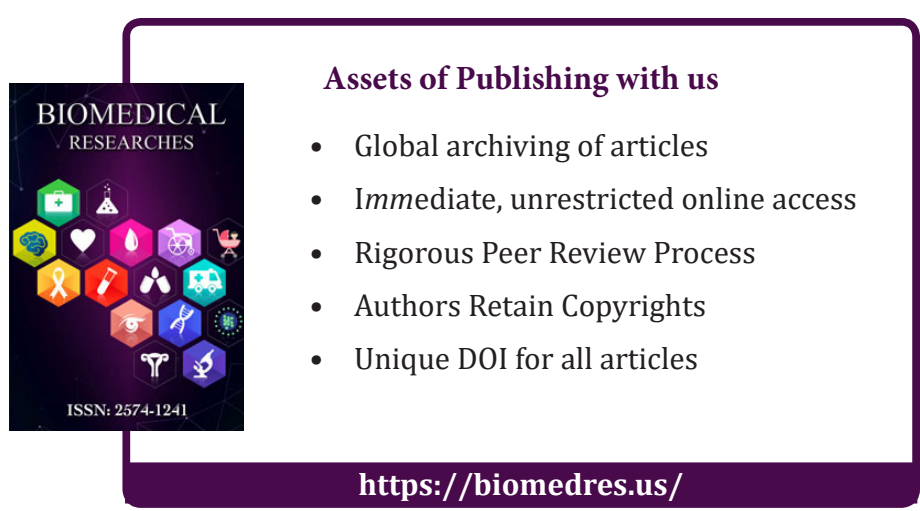

